\title{
Performance Characterization of Multiband UWB Communication Systems Using Poisson Cluster Arriving Fading Paths
}

\author{
W. Pam Siriwongpairat, Student Member, IEEE, Weifeng Su, Member, IEEE, and K. J. Ray Liu, Fellow, IEEE
}

\begin{abstract}
This paper provides a novel performance analysis for ultra-wideband (UWB) systems that successfully captures the unique multipath-rich property and multipath-clustering phenomenon of UWB channels. Using the Saleh-Valenzuela model, we characterize pairwise error probability and outage probability for UWB systems employing multiband OFDM based on the cluster arrival rate, the ray arrival rate within a cluster, and the cluster and ray decay factors. Furthermore, an approximation technique is established, which allows us to obtain closed-form performance formulations that provide insightful understanding of the effect of channel characteristics on the performances of UWB systems. Finally, we characterize the effect of random-clustering phenomenon on the performance of UWB-multiple-input-multiple-output systems. The theoretical results reveal that regardless of the clustering behavior of UWB channels, the diversity gain can be improved by increasing the number of jointly encoded subcarriers, the number of jointly encoded orthogonal frequency-division multiplexing symbols, or the number of antennas. The coding gain on the other hand, depends heavily on the cluster-arriving channels. Extensive simulation results are provided to support the theoretical analysis.
\end{abstract}

Index Terms-Multiband orthogonal frequency-division multiplexing (OFDM), multiple antennas, ultra-wideband (UWB).

\section{INTRODUCTION}

$\mathbf{T}$ HE ultra-wide bandwidth of ultra-wideband (UWB) gives rise to important differences between UWB and narrowband channels, especially with respect to the number of resolvable paths and arrival times of multipath components [1]. In particular, the large bandwidth of UWB waveform considerably increases the ability of a receiver to resolve different reflections in UWB channels. As a result, the received signal contains a significant number of resolvable multipath components. Additionally, due to the fine time resolution of UWB waveform, the multipath components tend to occur in cluster rather than in a continuum, as is common for narrowband channels.

In recent years, performance analysis of UWB systems has been an area of considerable interest. There is a large number of

Manuscript received March 1, 2005; revised October 13, 2005. This work was supported in part by U.S. Army Research Laboratory under Cooperative Agreement DAAD 190120011.

W. P. Siriwongpairat and K. J. R. Liu are with the Department of Electrical and Computer Engineering and the Institute for Systems Research, University of Maryland, College Park, MD 20742 USA (e-mail: wipawee@eng.umd.edu; kjrliu@eng.umd.edu).

W. Su was with the Department of Electrical and Computer Engineering and the Institute for Systems Research, University of Maryland, College Park, MD 20742 USA. He is now with the Department of Electrical Engineering, State University of New York (SUNY) at Buffalo, Buffalo, NY 14260 USA (e-mail: weifeng@eng.buffalo.edu).

Digital Object Identifier 10.1109/JSAC.2005.863822 papers dealing with the performance of UWB systems over additive white Gaussian noise (AWGN), as well as fading channels (see [2]-[8] and references therein). Although a clustering phenomenon has been observed in several large data sets of UWB channel measurements [9], it has not been taken into consideration for the analysis due to the fact that random clustering behavior introduces the difficulty in evaluating the analytical performance. In fact, most of the existing works are based on the stochastic tapped-delay-line (STDL) models [10] used in conventional narrowband/wideband systems. However, performance analysis in STDL models is basically an extension of that for narrowband systems. More importantly, it does not reflect the random-clustering property of UWB channels.

In order to implement an efficient UWB system, it is vital to capture the behavior of UWB channels, which has been characterized by the Saleh-Valenzuela (S-V) model [11]. A major difference between the S-V model and the STDL channel models is that in S-V model, the multipath components randomly arrive in cluster, and the arrival times are modeled by Poisson process. The multipath arrivals are grouped into cluster arrivals and ray arrivals within each cluster. The $\mathrm{S}-\mathrm{V}$ model is shown by the IEEE 802.15.3a Task Group [9] to best fit the realistic UWB channel measurements.

In this paper, we analyze the performance of UWB multiband orthogonal frequency-division multiplexing (OFDM) systems [12] by taking into account the multipath-rich and clustering characteristics of UWB channels. Using the S-V model, we characterize the UWB performance in terms of cluster arrival rate, ray arrival rate, and cluster and ray decay factors. Pairwise error probability (PEP) and outage probability are used as our performance criteria. First, we provide an exact PEP formulation for single-antenna multiband UWB systems. Then, we establish an approximation approach, which allows us to obtain a closed-form PEP formulation and an explicit outage probability expression. Finally, we generalize the performance results to space-time-frequency (STF) coded UWB multiple-input-multiple-output (MIMO) systems. The theoretical analysis reveals that the coding gain severely rely on the clustering property of UWB channels, whereas the diversity gain is the same for any environment. Simulation results are provided to support the theoretical analysis.

\section{SYSTEM MODEL}

We consider a peer-to-peer multiband OFDM system [12] as proposed in the IEEE 802.15.3a standard [9]. The multiband approach divides the available UWB spectrum into several subbands. Within each subband, the data is modulated using 
OFDM with $N$ subcarriers. The modulated OFDM symbols can be time-interleaved across different subbands [12].

\section{A. Channel Model}

The channel model specified in the IEEE 802.15.3a standard [1] is based on the S-V model for indoor channels [11]. In S-V model, the channel impulse response can be modeled by

$$
h(t)=\sum_{c=0}^{C} \sum_{l=0}^{L} \alpha(c, l) \delta\left(t-T_{c}-\tau_{c, l}\right)
$$

where $\alpha(c, l)$ denotes the gain of the $l$ th multipath component in the $c$ th cluster, $T_{c}$ is the delay of the $c$ th cluster, and $\tau_{c, l}$ is the delay of the $l$ th path in the $c$ th cluster relative to the cluster arrival time. The cluster arrivals and the path arrivals within each cluster can be modeled as Poisson distribution with rate $\Lambda$ and rate $\lambda(\lambda>\Lambda)$, respectively. The multipath gain coefficients $\alpha(c, l)$ are modeled as zero-mean, complex Gaussian random variables with variances [1]

$$
\Omega_{c, l}=E\left[|\alpha(c, l)|^{2}\right]=\Omega_{0,0} e^{-T_{c} / \Gamma-\tau_{c, l} / \gamma}
$$

where $\Omega_{0,0}$ is the mean energy of the first path of the first cluster, $\Gamma$ is the cluster decay factor, and $\gamma$ is the ray decay factor. The powers of the multipath components are normalized such that $\sum_{c=0}^{C} \sum_{l=0}^{L} \Omega_{c, l}=1$.

\section{B. Signal Model}

At the transmitter, an information sequence is partitioned into blocks. Each block is mapped onto an $N \times 1$ matrix $\mathbf{D}=\left[\begin{array}{llll}d(0) & d(1) & \ldots & d(N-1)\end{array}\right]^{T}$, where $d(n)$, $n=0,1, \ldots, N-1$, represents the complex channel symbol to be transmitted over subcarrier $n$. Suppose the information is jointly encoded across $M(1 \leq M \leq N)$ subcarriers. Particularly, the matrix $\mathbf{D}$ is a concatenation of $P=\lfloor N / M\rfloor$ data matrices as follows:

$$
\mathbf{D}=\left[\begin{array}{lllll}
\mathbf{D}_{0}^{T} & \mathbf{D}_{1}^{T} & \ldots & \mathbf{D}_{(P-1)}^{T} & \mathbf{0}_{(N-P M) \times 1}
\end{array}\right]^{T}
$$

where $\mathbf{D}_{p}$ is an $M \times 1$ data matrix defined as $\mathbf{D}_{p}=$ $\left[\begin{array}{llll}d_{p}(0) & d_{p}(1) & \ldots & d_{p}(M-1)\end{array}\right]^{T}$ with $d_{p}(n) \triangleq d(p M+n)$ for $p=0,1, \ldots, P-1$, and $\mathbf{0}_{m \times n}$ is an $m \times n$ all-zero matrix. The data matrices $\mathbf{D}_{p}$ are independently designed for different $p$, and each data symbol $d_{p}(n)$ is normalized to have unit energy.

Assume that the cyclic prefix length is greater than the duration of the channel impulse response. The received signal at the $n$th subcarrier is given by

$$
y(n)=\sqrt{E_{s}} d(n) H(n)+z(n)
$$

where $E_{s}$ is the average transmitted energy per symbol, $H(n)$ is the channel frequency response, and $z(n)$ denotes the noise sample. We model $z(n)$ as zero-mean complex Gaussian random variable with variance $N_{0}$. In (4), $H(n)$ is specified as

$$
H(n)=\sum_{c=0}^{C} \sum_{l=0}^{L} \alpha(c, l) \exp \left(-\mathbf{j} 2 \pi n \Delta f\left(T_{c}+\tau_{c, l}\right)\right)
$$

where $\mathbf{j} \triangleq \sqrt{-1}, \Delta f=1 / T$ is the frequency separation between two adjacent subcarriers, and $T$ is the OFDM symbol period. We assume that $H(n)$ is known at the receiver, but not at the transmitter.

\section{Performance AnAlysis}

In this section, we characterize the PEP and outage probability of multiband UWB systems in terms of multipath arrival rates and decay factors using the $\mathrm{S}-\mathrm{V}$ fading model.

\section{A. Average PEP Analysis}

For subsequent performance evaluation, we format the received signal in (4) in a vector form as

$$
\mathbf{Y}_{p}=\sqrt{E_{s}} X\left(\mathbf{D}_{p}\right) \mathbf{H}_{p}+\mathbf{Z}_{p}
$$

where $X\left(\mathbf{D}_{p}\right)=\operatorname{diag}\left(d_{p}(0), d_{p}(1), \ldots, d_{p}(M-1)\right)$ is an $M \times M$ diagonal matrix with the elements of $\mathbf{D}_{p}$ on its main diagonal. In (6), the channel matrix $\mathbf{H}_{p}$, the received signal matrix $\mathbf{Y}_{p}$, and the noise matrix $\mathbf{Z}_{p}$ are of the same forms as $\mathbf{D}_{p}$ by replacing $d$ with $H, y$ and $z$, respectively. Assume that $E_{s}$ is known at the receiver. The receiver exploits a maximum-likelihood decoder, where the decoding process is jointly performed within each data matrix $\mathbf{D}_{p}$, and the decision rule is

$$
\hat{\mathbf{D}}_{p}=\arg \min _{\mathbf{D}_{p}}\left\|\mathbf{Y}_{p}-\sqrt{E_{s}} X\left(\mathbf{D}_{p}\right) \mathbf{H}_{p}\right\|^{2}
$$

where $\|\cdot\|$ denotes the Frobenius norm [13].

Suppose that $\mathbf{D}_{p}$ and $\hat{\mathbf{D}}_{p}$ are two distinct data matrices. Following the computation steps as in [10], the average PEP, denoted as $P_{e}$, is given by

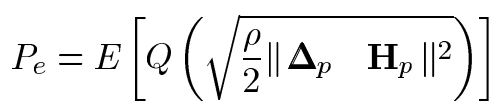

where $\rho=E_{s} / N_{0}$ is the average signal-to-noise ratio (SNR), $\boldsymbol{\Delta}_{p}=X\left(\mathbf{D}_{p}\right)-X\left(\hat{\mathbf{D}}_{p}\right)$, and $Q(x)$ is the Gaussian error function. Denoting

$$
\eta=\left\|\boldsymbol{\Delta}_{p} \quad \mathbf{H}_{p}\right\|^{2}
$$

and using an alternate representation of $Q$ function [14], $Q(x)=(1 / \pi) \int_{0}^{\pi / 2} \exp \left(-\left(x^{2} / 2 \sin ^{2} \theta\right)\right) d \theta$ for $x \geq 0$, the average PEP in (8) can be expressed as

$$
P_{e}=\frac{1}{\pi} \int_{0}^{\pi / 2} \mathcal{M}_{\eta}\left(-\frac{\rho}{4 \sin ^{2} \theta}\right) d \theta
$$

where $\mathcal{M}_{\eta}(s)=E[\exp (s \eta)]$ represents the moment generating function (MGF) of $\eta$ [14].

Observe that the variable $\eta$ depends on the path delays, as well as the multipath gains $\alpha(c, l)$ whose variances also depend on the path delays $T_{c}$ and $\tau_{c, l}$ which are based on Poisson process. In general, it is difficult, if not impossible, to determine $\mathcal{M}_{\eta}(s)$ in closed form. However, for an uncoded multiband UWB system, i.e., when the number of jointly encoded subcarriers is $M=1$, we have the following result.

Theorem 1: If there is no coding across subcarriers, the average PEP of a UWB multiband OFDM system is

$$
P_{e}=\frac{1}{\pi} \int_{0}^{\pi / 2}\left(1+\frac{\rho}{4 \sin ^{2} \theta}|d-\hat{d}|^{2}\right)^{-1} d \theta
$$

for any channel model parameters.

The proof of Theorem 1 is omitted due to space limitation. The reader can refer to [15] for further details. The above result reveals that the performance of an uncoded multiband UWB 
system does not depend on multipath arrival rates or decay factors, and it is the same as that of narrowband system in Rayleighfading environment [14]. This implies that we cannot gain from the rich multipath-clustering property of UWB channels if the data is not encoded across subcarriers.

\section{B. Approximate PEP Formulation}

We establish here an approximation which allows us to obtain an insightful understanding of the UWB systems when the information is jointly encoded across subcarriers.

According to ([16, p. 29]), the quadratic form in a zero-mean Gaussian random vector $\mathbf{x}=\left[x_{1}, \ldots, x_{M}\right]^{T}$ can be represented by a weighted summation of $\left|v_{n}\right|^{2}$, where $v_{n}$ are mutually independent standard Gaussian random variables, and the weights are the eigenvalues of the covariance matrix of $\mathbf{x}$. Observe from (9) that $\eta=\left(\boldsymbol{\Delta}_{p} \mathbf{H}_{p}\right)^{\mathcal{H}} \boldsymbol{\Delta}_{p} \mathbf{H}_{p}$ is in the quadratic form and $E\left[\boldsymbol{\Delta}_{p} \mathbf{H}_{p}\right]=\mathbf{0}$. Therefore, using the representation of the quadratic form in ([16, p. 29]), we have

$$
\eta \approx \sum_{n=1}^{M} \operatorname{eig}_{n}(\boldsymbol{\Phi})\left|\mu_{n}\right|^{2}
$$

where $\mu_{n}$ are independent identically distributed (i.i.d.) standard Gaussian random variables, and

$$
\boldsymbol{\Phi}=E\left[\boldsymbol{\Delta}_{p} \mathbf{H}_{p}\left(\boldsymbol{\Delta}_{p} \mathbf{H}_{p}\right)^{\mathcal{H}}\right]=\boldsymbol{\Delta}_{p} \mathbf{R}_{M} \boldsymbol{\Delta}_{p}^{\mathcal{H}}
$$

in which $\mathbf{R}_{M}=E\left[\mathbf{H}_{p} \mathbf{H}_{p}^{\mathcal{H}}\right]$ is an $M \times M$ correlation matrix. Let the eigenvalues $\operatorname{eig}_{n}(\boldsymbol{\Phi})$ be arranged in a nonincreasing order as: $\operatorname{eig}_{1}(\boldsymbol{\Phi}) \geq \operatorname{eig}_{2}(\boldsymbol{\Phi}) \cdots \geq \operatorname{eig}_{M}(\boldsymbol{\Phi})$. By Ostrowski's theorem ([13, p. 224]), the eigenvalues of $\Phi$ are given by

$$
\operatorname{eig}_{n}(\boldsymbol{\Phi})=\operatorname{eig}_{n}\left(\boldsymbol{\Delta}_{p} \mathbf{R}_{M} \boldsymbol{\Delta}_{p}^{\mathcal{H}}\right)=\nu_{n} \operatorname{eig}_{n}\left(\mathbf{R}_{M}\right)
$$

where $\nu_{n}$ is a nonnegative real number that satisfies $\operatorname{eig}_{M}\left(\Delta_{p} \Delta_{p}^{\mathcal{H}}\right) \leq \nu_{n} \leq \operatorname{eig}_{1}\left(\Delta_{p} \Delta_{p}^{\mathcal{H}}\right)$ for $n=1,2, \ldots, M$ From (12) and (14), we can approximate the MGF $\mathcal{M}_{\eta}(s)$ as

$$
\mathcal{M}_{\eta}(s) \approx \prod_{n=1}^{M} \frac{1}{1-s \nu_{n} \operatorname{eig}_{n}\left(\mathbf{R}_{M}\right)}
$$

Now, the remaining problem is to determine the matrix $\mathbf{R}_{M}$. We observe that the $\left(n, n^{\prime}\right)$ th entry of the matrix $\mathbf{R}_{M}$ is $E\left[H(n) H\left(n^{\prime}\right)^{*}\right]$ for $0 \leq n, n^{\prime} \leq M-1$. The elements on the main diagonal of $\mathbf{R}_{M}$ are given by

$$
R(n, n)=E\left[|H(n)|^{2}\right]=\sum_{c=0}^{C} \sum_{l=0}^{L} E\left[|\alpha(c, l)|^{2}\right]=1 .
$$

The off-diagonal elements of $\mathbf{R}_{M}, R\left(n, n^{\prime}\right)$ for $n \neq n^{\prime}$, can be evaluated as follows:

$R\left(n, n^{\prime}\right)=\sum_{c=0}^{C} \sum_{l=0}^{L} E\left[E\left[|\alpha(c, l)|^{2}\right] e^{-\mathbf{j} 2 \pi\left(n-n^{\prime}\right) \Delta f\left(T_{c}+\tau_{c, l}\right)}\right]$.

Let $R\left(n, n^{\prime}\right) \triangleq R\left(n-n^{\prime}\right)$, and substitute (2) into (17), yielding

$$
R(m)=\sum_{c=0}^{C} \sum_{l=0}^{L} \Omega_{0,0} G_{c, l}(m)
$$

where

$$
G_{c, l}(m)=E\left[\exp \left(-g\left(\frac{1}{\Gamma}, m\right) T_{c}-g\left(\frac{1}{\gamma}, m\right) \tau_{c, l}\right)\right]
$$

and $g(a, m) \triangleq a+\mathbf{j} 2 \pi m \Delta f$

To calculate $G_{c, l}(m)$, we denote $x_{i}$ as an interarrival time between clusters $i$ and $i-1$. From the Poisson distribution of the cluster delays, $x_{i}$ can be modeled as i.i.d. exponential random variables with parameter $\Lambda$, and the delay of the $c$ th cluster $T_{c}$ can be expressed as $T_{c}=\sum_{i=0}^{c} x_{i}$. Similarly, let $v_{c, j}$ denote an interarrival time between rays $j$ and $j-1$ in the $c$ th cluster. We can model $v_{c, j}$ as i.i.d. exponential random variables with parameter $\lambda$, and the delay $\tau_{c, l}=\sum_{j=0}^{l} v_{c, j}$. Rewriting $G_{c, l}(m)$ in terms of $x_{i}$ and $v_{c, j}$, (19) becomes

$$
\begin{aligned}
G_{c, l}(m) & =E\left[\prod_{i=0}^{c} e^{-g((1 / \Gamma), m) x_{i}}\right] E\left[\prod_{j=0}^{l} e^{-g((1 / \gamma), m) v_{c}, j}\right] \\
& =\left(\frac{\Lambda}{\Lambda+g\left(\frac{1}{\Gamma}, m\right)}\right)^{c}\left(\frac{\lambda}{\lambda+g\left(\frac{1}{\gamma}, m\right)}\right)^{l}
\end{aligned}
$$

Substitute (20) into (18), and use the fact that for UWB channels, the number of clusters $C$ and the number of rays $L$ are generally large. Then, we obtain

$$
R(m)=\Omega_{0,0} \frac{\Lambda+g\left(\frac{1}{\Gamma}, m\right)}{g\left(\frac{1}{\Gamma}, m\right)} \frac{\lambda+g\left(\frac{1}{\gamma}, m\right)}{g\left(\frac{1}{\gamma}, m\right)} .
$$

Finally, by substituting (15) into (10), we can characterize the multiband UWB performance as follows.

Theorem 2: If the information is jointly encoded across $M(1 \leq M \leq N)$ subcarriers, the average PEP of a multiband UWB system can be approximated as

$$
P_{e} \approx \frac{1}{\pi} \int_{0}^{\pi / 2} \prod_{n=1}^{M}\left(1+\frac{\rho \nu_{n}}{4 \sin ^{2} \theta} \operatorname{eig}_{n}\left(\mathbf{R}_{M}\right)\right)^{-1} d \theta
$$

where the $M \times M$ matrix $\mathbf{R}_{M}$ is given by

$$
\mathbf{R}_{M}=\left(\begin{array}{cccc}
1 & R(1)^{*} & \ldots & R(M-1)^{*} \\
R(1) & 1 & \ldots & R(M-2)^{*} \\
\vdots & \vdots & \ddots & \vdots \\
R(M-1) & R(M-2) & \ldots & 1
\end{array}\right)
$$

and $R(m)$ for $m=1,2, \ldots, M-1$ are defined in (21).

Note that the result in Theorem 2 can be straightforwardly extended to the case when interleaving or permutation over different subcarriers is applied. Specifically, if the data matrix is permuted such that the data symbol $d_{p}(n)$ is sent in subcarrier $\sigma_{p}(n)$ instead of subcarrier $n$, then the PEP of the permuted data matrix is of the same form as (22) with the off-diagonal elements of $\mathbf{R}_{M}$ replaced by $R\left(n, n^{\prime}\right)=R\left(\sigma_{p}(n)-\sigma_{p}\left(n^{\prime}\right)\right)$.

In the sequel, we discuss the approximate PEP in Theorem 2 for two special cases to get some insightful understanding. 
1) In case of no coding, i.e., $M=1$, the correlation matrix in (23) becomes $\mathbf{R}_{1}=1$, and $\nu_{1}=|d-\hat{d}|^{2}$. Thus, the PEP can be obtained from (22) as

$$
P_{e} \approx \frac{1}{\pi} \int_{0}^{\pi / 2}\left(1+\frac{\rho}{4 \sin ^{2} \theta}|d-\hat{d}|^{2}\right)^{-1} d \theta
$$

which is consistent with the exact PEP given in (11).

2) When the information is jointly encoded across two subcarriers, i.e., $M=2$, the eigenvalues of matrix $\mathbf{R}_{2}$ are $1+|R(1)|$ and $1-|R(1)|$. Substituting these eigenvalues into (22), we obtain the approximate PEP

$$
P_{e} \approx \frac{1}{\pi} \int_{0}^{\pi / 2}\left(1+\frac{\rho J+\rho^{2} \nu_{1} \nu_{2}\left(1-B^{2}\right)}{16 \sin ^{4} \theta}\right)^{-1} d \theta
$$

where $J=4 \sin ^{2} \theta\left[\nu_{1}+\nu_{2}+B\left(\nu_{1}-\nu_{2}\right)\right]$ and

$$
B=\Omega_{0,0} \frac{\left[\left(\Lambda+\frac{1}{\Gamma}\right)^{2}+b\right]^{1 / 2}\left[\left(\lambda+\frac{1}{\gamma}\right)^{2}+b\right]^{1 / 2}}{\left[\left(\frac{1}{\Gamma}\right)^{2}+b\right]^{1 / 2}\left[\left(\frac{1}{\gamma}\right)^{2}+b\right]^{1 / 2}}
$$

and $b=(2 \pi \Delta f)^{2}$. In UWB system, $b$ is normally much less than $\left(1 / \gamma^{2}\right)$ and $\left(1 / \Gamma^{2}\right)$. Hence, we have

$$
B \approx \Omega_{0,0}(\Lambda \Gamma+1)(\lambda \gamma+1) .
$$

The PEP in (22) reveals that the performance of coded multiband UWB system depends on the correlations in the frequency response among different subcarriers, which in turn relate to the path arrival rates and decay factors. If the number of jointly encoded subcarriers is $M=2,(25)$ shows that the UWB performance is related to the channel model $(\mathrm{CM})$ parameters through the factor $B$. In a short-range $(0-4 \mathrm{~m})$ line-of-sight environment, e.g., scenario for CM 1 [1], the product of the cluster arrival rate and cluster decay factor can be much less than one $(\Lambda \Gamma \ll 1)$. In such situation, (27) can be further simplified to $B \approx \Omega_{0,0}(\lambda \gamma+1)$, which implies that the performance severely depends only on the ray arrival rate and ray decay factor. The intuition behind this result is that when both cluster arrival rate and cluster decay factor are small, the effect of the first cluster will dominate. Hence, the performance can be approximated by taking into consideration only the first cluster. On the other hand, when both ray arrival rate and ray decay factor are small such that the product of these two parameters is much less than one $(\lambda \gamma \ll 1)$, (27) reduces to $B \approx \Omega_{0,0}(\Lambda \Gamma+1)$, which indicates that only the first path in each cluster seriously affects the performance.

For instance, suppose each data symbol $d$ is repeated in two subcarriers, denote $\nu=|d-\hat{d}|^{2}$, and let $\Delta f=4.125 \mathrm{MHz}$. From (25), the approximate PEP under CM 1 and CM 4 is:

- $\mathrm{CM} 1: \Lambda=0.023, \lambda=2.5, \Gamma=7.1, \gamma=4.3, B=0.985$

$$
P_{e} \approx \frac{1}{\pi} \int_{0}^{\pi / 2}\left(1+\frac{0.0294 \rho^{2} \nu^{2}}{16 \sin ^{4} \theta}\right)^{-1} d \theta .
$$

- $\mathrm{CM} 4: \Lambda=0.067, \lambda=2.1, \Gamma=24, \gamma=12, B=0.835$

$$
P_{e} \approx \frac{1}{\pi} \int_{0}^{\pi / 2}\left(1+\frac{0.3026 \rho^{2} \nu^{2}}{16 \sin ^{4} \theta}\right)^{-1} d \theta .
$$

We can see from the above examples that UWB performance in CM 4 is better than that in CM 1. This comes from the fact that the multipath components in CM 4 are more random than those in CM 1, which implies that compared with CM 1, CM 4 has less correlation in the frequency response among different subcarriers, and hence yields better performance.

\section{Outage Probability}

The outage probability [14] is defined as the probability that the combined SNR, $\zeta$, falls below a specified threshold, $\zeta_{o}$, namely $P_{\text {out }}=P\left(\zeta \leq \zeta_{o}\right)=\int_{0}^{\zeta_{o}} p_{\zeta}(x) d x$, where $p_{\zeta}(x)$ denotes the probability density function (PDF) of $\zeta$. Since the data is jointly en/decoded for each matrix $\mathbf{D}_{p}$, the combined SNR can be defined as

$$
\zeta=\frac{E_{s}\left\|X\left(\mathbf{D}_{p}\right) \mathbf{H}_{p}\right\|^{2}}{E\left[\left\|\mathbf{Z}_{p}\right\|^{2}\right]}=\frac{\rho}{M} \sum_{n=0}^{M-1}\left|H_{p}(n)\right|^{2}
$$

in which $\rho=E_{s} / N_{0}$. Denote $\xi=\sum_{n=0}^{M-1}\left|H_{p}(n)\right|^{2}$, then the outage probability can be expressed as

$$
P_{\text {out }}=P\left(\xi \leq \frac{M \zeta_{o}}{\rho}\right)=\int_{0}^{\left(M \zeta_{o} / \rho\right)} p_{\xi}(x) d x .
$$

To determine the PDF $p_{\xi}(x)$, we first obtain the MGF of $\xi$ from the MGF $\mathcal{M}_{\eta}(s)$ by replacing $\Delta_{p}$ with an identity matrix, and then apply the inverse Laplace transform to $\mathcal{M}_{\xi}(s)$. In case of no coding, we have $\mathcal{M}_{\xi}(s)=(1-s)^{-1}$, of which the corresponding PDF is $p_{\xi}(x)=\exp (-x)$ for $x \geq 0$ ([14, p. 22]). In case of jointly encoding across subcarriers, the $\operatorname{MGF} \mathcal{M}_{\xi}(s)$ can be obtained from (15) as

$$
\mathcal{M}_{\xi}(s) \approx \prod_{n=1}^{M} \frac{1}{1-\operatorname{seig}_{n}\left(\mathbf{R}_{M}\right)}=\sum_{n=1}^{M} \frac{A_{n}}{1-\operatorname{seig}_{n}\left(\mathbf{R}_{M}\right)}
$$

where the equality comes from the technique of partial fractions, $\mathbf{R}_{M}$ is defined in (23), and $A_{n}$ is given by

$$
A_{n}=\prod_{n^{\prime}=1, n^{\prime} \neq n}^{M} \frac{\operatorname{eig}_{n}\left(\mathbf{R}_{M}\right)}{\operatorname{eig}_{n}\left(\mathbf{R}_{M}\right)-\operatorname{eig}_{n^{\prime}}\left(\mathbf{R}_{M}\right)} .
$$

Applying the inverse Laplace transform to $\mathcal{M}_{\xi}(s)$, we have

$$
p_{\xi}(x) \approx \sum_{n=1}^{M} \frac{A_{n}}{\operatorname{eig}_{n}\left(\mathbf{R}_{M}\right)} \exp \left(-\frac{x}{\operatorname{eig}_{n}\left(\mathbf{R}_{M}\right)}\right), \quad x \geq 0 .
$$

Finally, substituting the above PDF $p_{\xi}(x)$ into (29) gives rise to the following results.

Theorem 3: If there is no coding across subcarriers, the outage probability of a multiband UWB system is given by

$$
P_{\text {out }}=1-\exp \left(-\frac{\zeta_{o}}{\rho}\right)
$$

for any channel parameters. In case of jointly encoding across $M(1<M \leq N)$ subcarriers, the outage probability is

$$
P_{\text {out }} \approx \sum_{n=1}^{M} A_{n}\left(1-\exp \left(-\frac{\zeta_{o} M}{\rho \operatorname{eig}_{n}\left(\mathbf{R}_{M}\right)}\right)\right)
$$

where $\mathbf{R}_{M}$ is specified in (23), and $A_{n}$ is defined in (30).

From the above analysis, we can see that the outage probability follows the same behaviors as the average PEP.

Specifically, (33) discloses that the outage probability is related to the eigenvalues of the correlation matrix $\mathbf{R}_{M}$, which depends on the path arrival rates and decay factors. As an example, 
consider the case of jointly encoding across $M=2$ subcarriers, then the outage probability can be approximated as

$$
P_{\mathrm{out}} \approx 1-v e^{-\left(2 \zeta_{\circ} / \rho(1+B)\right)}-v e^{-\left(2 \zeta_{o} / \rho(1-B)\right)}
$$

where $v=1+B^{-1} / 2$ and $B$ is defined in (34). Since $B$ takes any value between 0 and $1(0<B<1)$, the higher the $B$ the larger the outage probability $P_{\text {out }}$ in (34). For instance, $B=0.9852$ in case of $\mathrm{CM} 1$ and $B=0.8351$ in case of CM 4. When the SNR $\rho$ is small, $\exp \left(-\left(2 \zeta_{o} / \rho(1+B)\right)\right) \gg$ $\exp \left(-\left(2 \zeta_{o} / \rho(1-B)\right)\right)$, and hence the third term in (34) is negligible. Then, the outage probability can be approximated by (32), which implies that in low SNR region we cannot gain from the multipath-clustering property of UWB channel. As the SNR increases, $P_{\text {out }}$ for CM 4 drops faster than that for CM 1 due to the effect of the third term in $P_{\text {out }}$ expressions. Explicitly, the term $0.0987 \exp \left(-\left(12.131 \zeta_{o} / \rho\right)\right)$ for $\mathrm{CM} 4$ increases with SNR $\rho$ much faster than the term $0.0075 \exp \left(-\left(134.91 \zeta_{o} / \rho\right)\right)$ for CM 1. Hence, the performance for CM 4 tends to be better than that for CM 1 at high SNR.

\section{ANALYSIS FOR UWB-MIMO SYSTEMS}

We briefly describe a UWB-MIMO system model, and then apply the proposed approximation technique to characterize PEP performances of multiband UWB-MIMO system.

\section{A. UWB-MIMO System Model}

Consider a UWB-MIMO system with $N_{t}$ transmit and $N_{r}$ receive antennas. The channel impulse response from the $i$ th transmit antenna to the $j$ th receive antenna during the $k$ th OFDM block can be modeled in the same form as (1) with the multipath gain coefficient replaced by $\alpha_{i j}^{k}(c, l)$. We assume that the average powers $\Omega_{c, l}$ and the delays $T_{c}$ and $\tau_{c, l}$ are the same for every transmit-receive link.

At the transmitter, the information is jointly encoded across $N_{t}$ transmit antennas, $M$ OFDM subcarriers, and $K$ OFDM blocks. Each STF codeword can be expressed as a $K M \times N_{t}$ matrix $\mathbf{D}_{p}=\left[\begin{array}{lll}\left(\mathbf{D}_{p}^{0}\right)^{T}\left(\mathbf{D}_{p}^{1}\right)^{T} \ldots & \left(\mathbf{D}_{p}^{K-1}\right)^{T}\end{array}\right]^{T}$, where $\mathbf{D}_{p}^{k}=\left[\begin{array}{llll}\mathbf{d}_{p, 1}^{k} & \mathbf{d}_{p, 2}^{k} & \ldots & \mathbf{d}_{p, N_{t}}^{k}\end{array}\right]$ and $\mathbf{d}_{p, i}^{k}=$ $\left[d_{i}^{k}(p M) d_{i}^{k}(p M+1) \ldots d_{i}^{k}(p M+M-1)\right]^{T}$ for $i=$ $1,2, \ldots, N_{t}$ and $k=0,1, \ldots, K-1$. The symbol $d_{i}^{k}(n)$, $n=0,1, \ldots, N$, represents the complex symbol to be transmitted over subcarrier $n$ by transmit antenna $i$ during the $k$ th OFDM symbol period.

The received signal at the $n$th subcarrier at receive antenna $j$ during the $k$ th OFDM symbol duration can be expressed as

$$
y_{j}^{k}(n)=\sqrt{\frac{E_{s}}{N_{t}}} \sum_{i=1}^{N_{t}} d_{i}^{k}(n) H_{i j}^{k}(n)+z_{j}^{k}(n)
$$

where $H_{i j}^{k}(n)=\sum_{c=0}^{C} \sum_{l=0}^{L} \alpha_{i j}^{k}(c, l) e^{-\mathbf{j} 2 \pi n \Delta f\left(T_{c}+\tau_{c, l}\right)}$ is the channel frequency response, and $z_{j}^{k}(n)$ is the additive noise. The receiver exploits a maximum likelihood decoder, where the decoding is jointly performed across $N_{r}$ receive antennas.

Due to the band hopping, the $K$ OFDM symbols in each STF codeword are sent over different subbands. With an ideal band hopping, we assume that the signals transmitted over $K$ different subbands undergo independent fading. For simplicity, the MIMO channel is assumed to be spatially uncorrelated.

\section{B. Pairwise Error Probability}

Similarly, the PEP between two distinct STF codewords $\mathbf{D}_{p}$ and $\hat{\mathbf{D}}_{p}$ can be given by

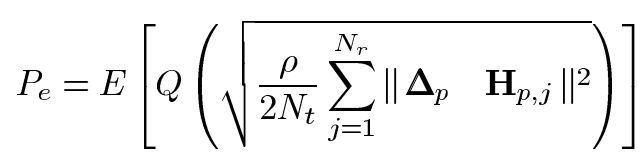

where $\boldsymbol{\Delta}_{p}=X\left(\mathbf{D}_{p}\right)-X\left(\hat{\mathbf{D}}_{p}\right)$, and $X\left(\mathbf{D}_{p}\right)$ converts each column of $\mathbf{D}_{p}=\left[\begin{array}{lll}\mathbf{d}_{p, 1} & \ldots & \mathbf{d}_{p, N_{t}}\end{array}\right]$ into a diagonal matrix, resulting in an $K M \times K M N_{t}$ matrix: $X\left(\mathbf{D}_{p}\right)=\left[\begin{array}{llll}\operatorname{diag}\left(\mathbf{d}_{p, 1}\right) & \ldots & \operatorname{diag}\left(\mathbf{d}_{p, N_{t}}\right)\end{array}\right] . \operatorname{In}(36), \mathbf{H}_{p, j}=$ $\left[\begin{array}{llll}\mathbf{H}_{p, 1 j}^{T} & \mathbf{H}_{p, 2 j}^{T} & \ldots & \mathbf{H}_{p, N_{t} j}^{T}\end{array}\right]^{T}$ is $K M N_{t} \times 1$ channel matrix, in which $\mathbf{H}_{p, i j}={ }^{p} H_{i j}^{0}(p M) \cdots H_{i j}^{0}(p M+M-1)$ $\left.\cdots H_{i j}^{K-1}(p M) \cdots H_{i j}^{K-1}(p M+M-1)\right]^{T}$. Following the same procedure as in single antenna case, we first obtain

$$
\eta=\sum_{j=1}^{N_{r}}\left\|\boldsymbol{\Delta}_{p} \quad \mathbf{H}_{p}\right\|^{2} \approx \sum_{j=1}^{N_{r}} \sum_{n=1}^{M} \operatorname{eig}_{n}\left(\Phi_{j}\right)\left|\mu_{j, n}\right|^{2}
$$

where $\boldsymbol{\Phi}_{j}=\boldsymbol{\Delta}_{p} E\left[\mathbf{H}_{p, j} \mathbf{H}_{p, j}^{\mathcal{H}}\right] \boldsymbol{\Delta}_{p}^{\mathcal{H}}$, and $\mu_{j, n}$ are i.i.d. zeromean Gaussian random variables with unit variance. After some algebra, $\boldsymbol{\Phi}_{j}$ can be re-expressed as

$$
\boldsymbol{\Phi}_{j}=\left(\mathbf{D}_{p}-\hat{\mathbf{D}}_{p}\right)\left(\mathbf{D}_{p}-\hat{\mathbf{D}}_{p}\right)^{\mathcal{H}} \circ\left(\mathbf{I}_{K} \otimes \mathbf{R}_{M}\right)
$$

where $\mathbf{R}_{M}$ is specified in (23), $\otimes$ denotes the Kronecker product, and $\circ$ denotes the Hadamard product [13]. Define $\mathbf{S}_{k} \triangleq\left(\mathbf{D}_{p}^{k}-\hat{\mathbf{D}}_{p}^{k}\right)\left(\mathbf{D}_{p}^{k}-\hat{\mathbf{D}}_{p}^{k}\right)^{\mathcal{H}}$. Suppose the information is repeated over $K$ OFDM symbols, then $\mathbf{S}_{k}$ is the same for all $k$. Substitute (38) into (37) and use the MGF of $\eta$, then we can approximate the average PEP of UWB-MIMO system as

$$
P_{e} \approx \frac{1}{\pi} \int_{0}^{\pi / 2} \prod_{n=1}^{M}\left(1+\frac{\rho \operatorname{eig}_{n}\left(\mathbf{S}_{0} \circ \mathbf{R}_{M}\right)}{4 N_{t} \sin ^{2} \theta}\right)^{-K N_{r}} d \theta .
$$

At high SNR, the PEP in (39) can be upper bounded as

$$
P_{e} \lesssim \prod_{n=1}^{r}\left(\frac{\rho}{4 N_{t}} \operatorname{eig}_{n}\left(\mathbf{S}_{0} \circ \mathbf{R}_{M}\right)\right)^{-K N_{r}}
$$

which implies a diversity order of $r K N_{r}$ and a coding gain of $\left(1 / 4 N_{t}\right)\left(\prod_{n=1}^{r} \operatorname{eig}_{n}\left(\mathbf{S}_{0} \circ \mathbf{R}_{M}\right)\right)^{1 / r}$, where $r$ denotes the rank of matrix $\mathbf{S}_{0} \circ \mathbf{R}_{M}$. Since UWB channel contains a large number of resolvable paths, $\mathbf{R}_{M}$ is generally of full rank. This leads to an interesting observation that the multiband UWB system achieves the same diversity advantage in different channel environment. Only the system coding gain that depends heavily on the cluster arriving fading paths.

To get some insight, consider the STF codeword based on a repetition code [17] with Alamouti's structure [18]. We have

$$
P_{e} \approx \frac{1}{\pi} \int_{0}^{\pi / 2} \prod_{n=1}^{M / 2}\left(1+\frac{\rho \nu}{8 \sin ^{2} \theta} \operatorname{eig}_{n}\left(\mathbf{R}_{(M / 2)}\right)\right)^{-2 K N_{r}} d \theta
$$

where $\nu \triangleq \sum_{i=1}^{2}\left|d_{i}-\hat{d}_{i}\right|^{2}$. For example, if $N_{t}=2, M=4$, then (41) can be upper bounded at high SNR as

$$
P_{e} \lesssim\left(\frac{\rho \nu}{8} \sqrt{1-\Omega_{0,0}^{2}(\Lambda \Gamma+1)^{2}(\lambda \gamma+1)^{2}}\right)^{-4 K N_{r}} .
$$

Here, the diversity gain is $4 K N_{r}$ for any channel parameters, while coding gain is $0.021 \nu$ for $\mathrm{CM} 1$ and $0.069 \nu$ for $\mathrm{CM} 4$. 


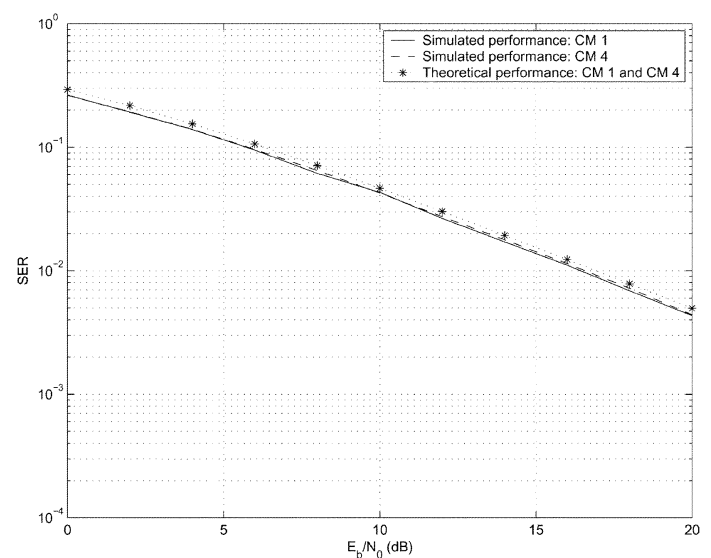

(a)

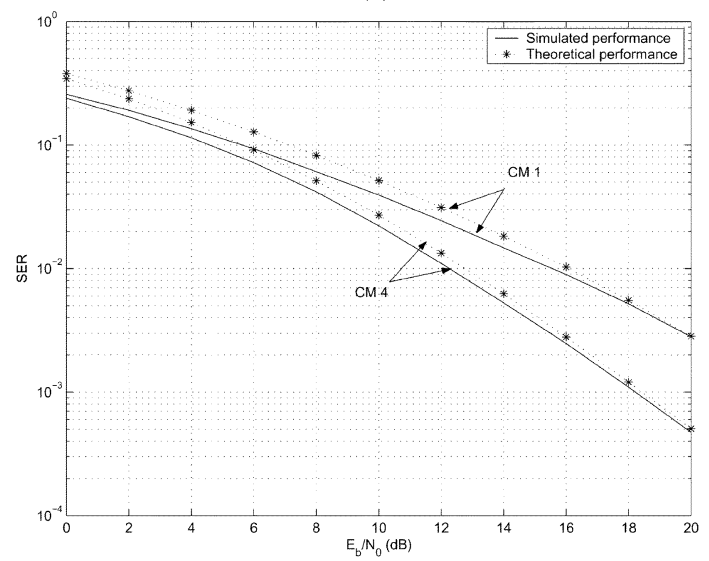

(b)

Fig. 1. PEP of multiband UWB-MIMO system. (a) No coding across subcarriers. (b) Jointly encoding across two subcarriers.

\section{Simulation Results}

We performed simulations for a multiband UWB system with $N=128$ subcarriers and the subband bandwidth of $528 \mathrm{MHz}$. The channel model parameters followed those for CM 1 and CM 4 [1]. The data symbols are selected from quadrature phaseshift keying (QPSK) constellations. The data matrix D in (3) were constructed via a repetition mapping [17], and Alamouti's structure was used for UWB-MIMO system.

Fig. 1(a) and (b) depict the average symbol-error-rate (SER) performances of single-antenna multiband UWB system as functions of average SNR per bit $\left(E_{b} / N_{0}\right)$ in decibels $(\mathrm{dB})$. The union bound [14] is used to obtain the average SER from the PEP formulation. In Fig. 1(a), we show the simulated and theoretical performances of uncoded multiband UWB system $(M=1)$. We observe that the performances of UWB system in CM 1 and CM 4 are almost the same, and they are close to the exact PEP calculation in (11). Simulation results confirm the theoretical expectation that the performance of uncoded multiband UWB systems is the same for every environment. Fig. 1(b) shows the performance in case of jointly encoding across two subcarriers $(M=2)$. The theoretical approximation obtained from (22) are close to the simulated performance for both CM 1 and CM 4. In addition, the performance obtained under CM 4 is superior to that under CM 1, which is in agreement with the theoretical results in Section III-B.

Fig. 2(a) and (b) plot the outage probability $P_{\text {out }}$ versus normalized average SNR $\rho / \zeta_{o}$ in $\mathrm{dB}$. We can observe that the

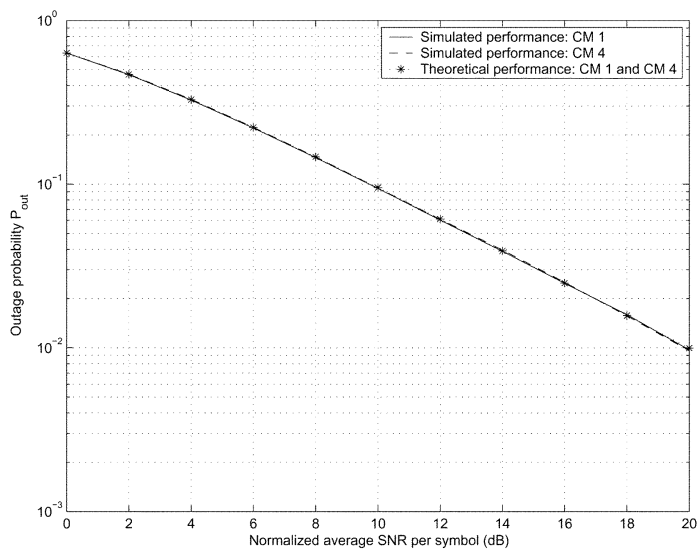

(a)

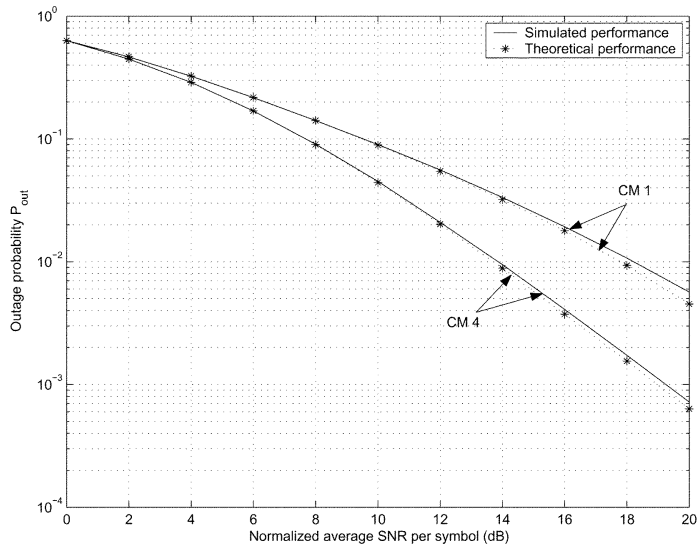

(b)

Fig. 2. Outage probability of multiband UWB system. (a) No coding across subcarriers. (b) Jointly encoding across two subcarriers.

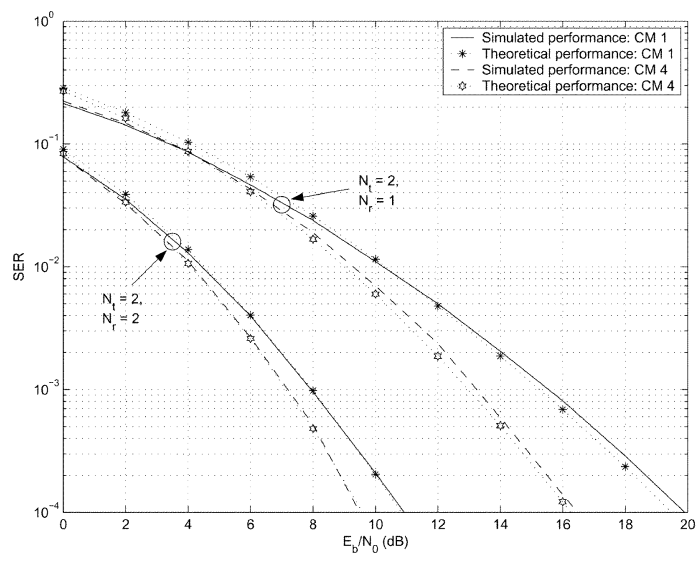

Fig. 3. PEP of multiband UWB-MIMO system with two transmit antennas and data jointly across four subcarriers.

outage probability follows the same tendencies as the average SER. The uncoded system yields the same outage probability in both CM 1 and CM 4, whereas the coded system under CM 4 achieves a lower outage probability, hence better performance, than that with CM 1.

Fig. 3 depicts the SER performances for UWB-MIMO system in case of jointly encoding across $N_{t}=2$ transmit antennas, $K=1$ OFDM symbol, and $M=4$ subcarriers. The theoretical SER was obtained from the union bound of the PEP formulation in (40). From both figures, we can see that the theoretical approximation in (40) correctly predicts the diversity and 
coding gains. Fig. 3 confirms our observation in Section IV that the multiband system under CM 4 outperforms that under CM 1 due to the larger coding gain.

\section{CONCLUSION}

In this paper, we provide PEP and outage probability analysis that captures the unique multipath-rich and random-clustering characteristics of UWB channels. Both theoretical and simulation results reveal that the performances of uncoded multiband UWB systems do not depend on the clustering property. In case of jointly encoding across subcarriers, theoretical approximations reveal that UWB performances depend heavily the cluster property of UWB channels. In case of jointly coding across two subcarriers, we can draw some interesting conclusions as follows. When the product of the cluster arrival rate and cluster decay factor is small, e.g., in a short-range $(0-4 \mathrm{~m})$ line-of-sight scenario, the effect of the first cluster will dominate and the UWB performance can be well approximated by taking into consideration only the first cluster. In contrast, when the product of the ray arrival rate and ray decay factor is much less than one, the performance seriously depends only on the first path in each cluster. Furthermore, we extend the analysis to that for UWB-MIMO systems. It turns out that the coding gain strongly relates to the channel model parameters whereas the diversity gain is the same for any channel environment.

\section{REFERENCES}

[1] J. Foerster et al., "Channel Modeling Sub-Committee Report Final," IEEE, IEEE 802.15-02/490, 2003.

[2] J. D. Choi and W. E. Stark, "Performance of ultra-wideband communications with suboptimal receivers in multipath channels," IEEE J. Sel. Areas Commun., vol. 20, no. 9, pp. 1754-1766, Dec. 2002.

[3] G. Durisi and S. Benedetto, "Performance evaluation of TH-PPM UWB systems in the presence of multiuser interference," IEEE Commun. Lett., vol. 7, no. 5, pp. 224-226, May 2003.

[4] N. Boubaker and K. B. Letaief, "Performance analysis of DS-UWB multiple access under imperfect power control," IEEE Trans. Commun., vol. 52, no. 9, pp. 1459-1463, Sep. 2004

[5] J. Zhang, R. A. Kennedy, and T. D. Abhayapala, "Performance of RAKE reception for ultra wideband signals in a lognormal fading channel," Int. J. Wireless Information Netw., pp. 193-200, Oct. 2005.

[6] W. P. Siriwongpairat, M. Olfat, and K. J. R. Liu, "Performance analysis and comparison of time hopping and direct sequence UWB-MIMO systems," EURASIP J. on Appl. Signal Proc. (Special Issue on UWB-State of the Art), no. 3, pp. 328-345, Mar. 2005.

[7] T. Kaiser, Ed., UWB Communications Systems-A Comprehensive Overview. ser. EURASIP Series on Signal Processing and Communications. Cairo, Egype: Hindawi, 2005.

[8] W. P. Siriwongpairat, W. Su, M. Olfat, and K. J. R. Liu, "Space-time-frequency coded multiband UWB communication systems," in Proc. IEEE Wireless Commun. Netw. Conf. (WCNC), vol. 1, Mar. 2005, pp. 426-431.

[9] IEEE 802.15WPAN High Rate Alternative PHY Task Group 3a (TG3a). [Online]. Available: www.ieee802.org/15/pub/ TG3a.html

[10] J. G. Proakis, Digital Communications, 4th ed. New York: McGrawHill, 2001.

[11] A. A. M. Saleh and R. A. Valenzuela, "A statistical model for indoor multipath propagation," IEEE J. Sel. Areas Commun., vol. 5, no. 2, pp. 128-137, Feb. 1987.

[12] A. Batra et al., "Design of a multiband OFDM system for realistic UWB channel environments," IEEE Trans. Microw. Theory Tech., vol. 52, no. 9, pp. 2123-2138, Sep. 2004

[13] R. A. Horn and C. R. Johnson, Matrix Analysis. Cambridge, U.K.: Cambridge Univ. Press, 1985.

[14] M. K. Simon and M. S. Alouini, Digital Communication Over Fading Channels: A Unified Approach to Performance Analysis. New York: Wiley, 2000.
[15] W. P. Siriwongpairat, W. Su, and K. J. R. Liu, "Characterizing performance of multiband UWB systems using Poisson Cluster arriving fading paths," in Proc. IEEE Workshop on Signal Process. Advances in Wireless Commun. (SPAWC), Jul. 2005, pp. 264-268.

[16] A. M. Mathai and S. B. Provost, Quadratic Forms in Random Variables: Theory and Applications. New York: Marcel Dekker, 1992.

[17] W. Su, Z. Safar, and K. J. R. Liu, "Toward maximum achievable diversity in space, time and frequency: Performance analysis and code design," IEEE Trans. Wireless Commun., vol. 4, no. 4, pp. 1847-1857, Jul. 2005.

[18] S. Alamouti, "A simple transmit diversity technique for wireless communications," IEEE J. Sel. Areas Commun., vol. 16, no. 8, pp. 1451-1458, Oct. 1998.

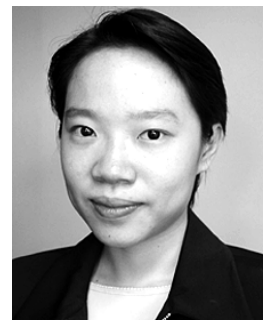

W. Pam Siriwongpairat (S'03-M'06) received the B.S. degree in electrical engineering from the Chulalongkorn University, Bangkok, Thailand, in 1999, and the M.S. and Ph.D. degree both in electrical engineering from the University of Maryland, College Park, in 2001 and 2005, respectively.

She is currently a Postdoctoral Research Associate with the Department of Electrical and Computer Engineering and the Institute for Systems Research (ISR), University of Maryland. Her current research interests include signal processing, wireless communications and networking, cross-layer design, and cooperative communications, with particular focus on ultra-wideband communications. Her research contributions encompass space-time and space-frequency coding for multiantenna ultra-wideband systems.

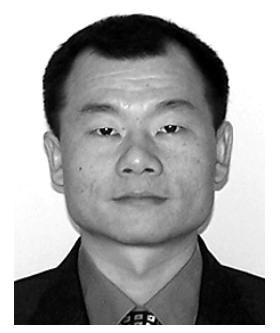

Weifeng Su (M'03) received the Ph.D. degree in electrical engineering from the University of Delaware, Newark, in 2002, and the B.S. and Ph.D. degrees in mathematics from Nankai University, Tianjin, China, in 1994 and 1999, respectively.

$\mathrm{He}$ is an Assistant Professor at the Department of Electrical Engineering, State University of New York (SUNY) at Buffalo. From June 2002 to March 2005, he was a Postdoctoral Research Associate with the Department of Electrical and Computer Engineering and the Institute for Systems Research (ISR), University of Maryland, College Park. His research interests span a broad range of areas from signal processing to wireless communications and networking, including space-time coding and modulation for MIMO wireless communications, MIMO-OFDM systems, cooperative communications for wireless networks, and ultra-wideband (UWB) communications.

Dr. Su received the Signal Processing and Communications Faculty Award from the University of Delaware in 2002 as an Outstanding Graduate Student in the field of signal processing and communications. In 2005, he received the Invention of the Year Award from the University of Maryland.

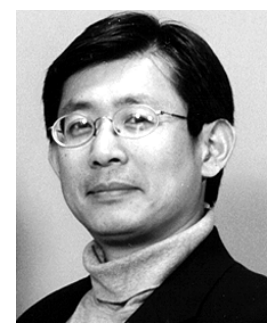

K. J. Ray Liu (F'03) received the B.S. degree from the National Taiwan University, Taipei, in 1983, and the Ph.D. degree from the University of California at Los Angeles (UCLA) in 1990, both in electrical engineering.

$\mathrm{He}$ is Professor and Director of Communications and Signal Processing Laboratories of Electrical and Computer Engineering Department and Institute for Systems Research, University of Maryland. His research contributions encompass broad aspects of wireless communications and networking, information forensics and security, multimedia communications and signal processing, bioinformatics and biomedical imaging, and signal processing algorithms and architectures.

Dr. Liu is the recipient of numerous honors and awards including best paper awards from IEEE Signal Processing Society (twice), IEEE Vehicular Technology Society, and EURASIP; IEEE Signal Processing Society Distinguished Lecturer, EURASIP Meritorious Service Award, and the National Science Foundation Young Investigator Award. He also received Poole and Kent Company Senior Faculty Teaching Award from A. James Clark School of Engineering, and Invention of the Year Award, both from University of Maryland. He is Vice President-Publication and on the Board of Governor of IEEE Signal Processing Society. He was the Editor-in-Chief of the IEEE Signal Processing Magazine, and was the founding Editor-in-Chief of the EURASIP Journal on Applied Signal Processing. 